\title{
Gauge-invariant theory for semiclassical magnetotransport through ballistic microstructures
}

\author{
Ludger Wirtz, Jian-Zhi Tang, and Joachim Burgdörfer \\ Department of Physics, University of Tennessee, Knoxville, Tennessee 37996-1200; \\ Oak Ridge National Laboratory, Oak Ridge, Tennessee 37831-6377; \\ and Institut für Theoretische Physik, Technische Universität Wien, Wiedner Hauptstrasse 8-10, A-1040 Wien, Austria
}

(Received 6 July 1998)

\begin{abstract}
Within the semiclassical theory of magnetotransport through ballistic cavities, fluctuations in the transmission amplitude and in the conductance originate from the Aharonov-Bohm phase of directed areas. We formulate the semiclassical transmission amplitude in gauge-invariant form. The gauge invariant phases can be visualized in terms of areas enclosed by classical paths, which consist of the real path connecting the entrance point to the exit point and a virtual path leading back to the entrance point. We implement this method on different levels of a semiclassical description of magnetotransport with applications to magnetoconductance fluctuations and correlations. The validity of the semiclassical theories is analyzed. [S0163-1829(99)10903-2]
\end{abstract}

\section{INTRODUCTION}

In recent years considerable effort has been made to describe electron transport through open ballistic microstructures for which the elastic and inelastic mean free paths are large compared to the system dimensions. ${ }^{1}$ Several experiments have been performed, showing strong fluctuations of the conductance as a function of a perpendicular homogeneous magnetic field. Investigations of magnetoconductance fluctuations have become a focal point for studies of regular and chaotic dynamics on a microscopic scale. ${ }^{2-6}$ A relation between periodic conductance fluctuations and scars in the wave functions has been pointed out. ${ }^{7,8}$

Quantum mechanically, ballistic transport through the microstructure represents a two-dimensional phase-coherent scattering problem of electrons entering through the entrance lead and scattering at the potential given by the walls of the structure before exiting through the same lead (reflection) or another lead (transmission). Classically, the electron is launched through the entrance lead into the cavity and undergoes multiple specular reflections at the walls before exiting. Semiclassical theory provides a link between the classical and the quantum description of ballistic transport. It is particularly useful in the study of "quantum chaos",9 and is well suited to explore nonuniversal properties of conductance fluctuations that originate from geometry-specific scattering processes. Several semiclassical approaches have been applied to the open billiard problem. ${ }^{10-19}$ While significant progress has been made, there remain considerable obstacles to a consistent semiclassical description of ballistic quantum transport in the presence of a magnetic field, some of which are the subject of this paper.

The semiclassical theory expresses the conductance in terms of a sum over classical paths connecting the entrance and the exit leads (or quantum wires), each path carrying an amplitude and a phase. The contribution of the phase that depends on the magnetic field $B$ (equivalent to an AharonovBohm phase), $\Phi_{B}=a B / c$, is determined by the directed area enclosed by the trajectory. The area $a$ is usually defined as the path integral over the vector potential $\vec{A}:{ }^{12,13}$

$$
a=\frac{1}{B} \int_{q} \vec{A} d \vec{r}
$$

where $q$ is the classical path starting at the entrance lead, bouncing with the cavity walls, and then exiting through the exit lead. It is important to note that the area as defined by Eq. (1) is not invariant under gauge transformations of the vector potential since $q$ is not a closed path. This results in a gauge dependence of transport properties that are shape specific. One purpose of the present paper is to formulate the semiclassical theory in gauge-invariant form. This is achieved by incorporating the proper magnetic phases into the wave function in the quantum leads. Semiclassically, this can be interpreted as adding a virtual path to the real path $q$ to form a closed path.

The quantum transport problem is characterized by different length scales, as given by the linear dimension of the cavity $D \approx \sqrt{A}$, where $A$ is the area of the billiard, and the width of the quantum wire $d$. In typical experimental realizations, the de Broglie wavelength of the electron $\lambda$ is small compared to the size of the structure $\lambda \ll D$ but comparable to the width of the quantum wire $\lambda \leqslant d$. This leads to diffractive scattering at the lead mouths that imposes limitations on the validity of a semiclassical theory. ${ }^{17-19}$ We compare different methods to overcome this problem and present results for magnetoconductance fluctuations and its autocorrelation function using different semiclassical approximations. Semiclassical results will be compared with full quantum calculations and experimental data. Furthermore, the significance of these results is analyzed in light of the observation that semiclassical theory violates unitarity. Deviations from unitarity as a function of the wave number and the parameters of the billiards will be investigated.

\section{GAUGE-INVARIANT SEMICLASSICAL THEORY}

We consider the ballistic transport through open quantum billiards with two leads, also referred to as quantum wires, which are attached to electron reservoirs at different electrochemical potentials. Within the framework of the Landauer formula ${ }^{20}$ the conductance 


$$
g=\sum_{m=1}^{N} g^{(m)}=\frac{2 e^{2}}{h} \sum_{m, n=1}^{N}\left|t_{m n}\right|^{2}
$$

is directly related to the transmission amplitudes $t_{m n}$ connecting the incoming mode $m$ in the quantum wire 1 (entrance lead) with the outgoing mode $n$ in the quantum wire 2 (exit lead). Each mode corresponds to a bound state in the coordinate transverse to the flux direction. The corresponding transverse wave function in the lead with infinitely highpotential walls in the absence of a magnetic field is

$$
\phi_{n}(y)=\sqrt{\frac{2}{d}} \sin \left[\frac{n \pi}{d}\left(y+\frac{d}{2}\right)\right]
$$

where $d$ is the width of the lead. $N$ is the total number of transmitted modes, while mode numbers $n>N$ correspond to evanescent waves.

The starting point of the semiclassical analysis is the quantum-mechanical expression for the transmission amplitude as a projection of the retarded Green's function $G$ onto the transverse wave functions in the leads: ${ }^{11}$

$$
\begin{aligned}
t_{m n}\left(k_{F}\right)= & -i \sqrt{v_{x_{2}, n} v_{x_{1}, m}} \\
& \times \int_{-d / 2}^{d / 2} d y_{2} \int_{-d / 2}^{d / 2} d y_{1} \phi_{n}^{*}\left(y_{2}\right) G\left(y_{1}, y_{2}, k_{F}\right) \phi_{m}\left(y_{1}\right) .
\end{aligned}
$$

The Green's function describes the constant energy propagation from the transverse coordinate $y_{1}$ in the mouth of the entrance lead to the transverse coordinate $y_{2}$ in the mouth of the exit lead and $v_{x_{1}, m}, v_{x_{2}, n}$ are the longitudinal velocities in the leads corresponding to modes $m$ and $n$. For simplicity the longitudinal coordinates $x_{1,2}$ of the entrance and exit lead mouths are suppressed. Atomic units $\left(\hbar=|e|=m_{e f f}=1\right)$ will be used throughout the paper. A more general expression for the multilead case has been given by Baranger and Stone ${ }^{21}$ where gauge invariance is discussed within the framework of quantum transport.

The semiclassical approximation proceeds by replacing the quantum-mechanical Green's function by its semiclassical limit $G^{S C}:{ }^{11,22}$

$$
\begin{array}{r}
G^{S C}\left(y_{1}, y_{2}, k_{F}\right)=\frac{2 \pi}{(2 \pi i)^{3 / 2}} \sum_{q: y_{1} \rightarrow y_{2}}\left|D_{q}\left(y_{1}, y_{2}, k_{F}\right)\right|^{1 / 2} \\
\quad \times \exp \left[i S_{q}\left(y_{1}, y_{2}, k_{F}\right)-i \frac{\pi}{2} \mu_{q}\right] .
\end{array}
$$

The summation extends over all classical paths $q$ connecting $y_{1}$ in the entrance lead with $y_{2}$ in the exit lead mouth. $S_{q}$ $=\int_{q} d \vec{r} \vec{p}$ is the action of the path, $\left|D_{q}\right|$ $=\left|\partial^{2} S_{q} / \partial y_{2} \partial y_{1}\right| /\left|v_{x_{1}} v_{x_{2}}\right|$ is a measure for the divergence of nearby trajectories, and $\mu_{q}$ is the Maslov index.

The homogeneous magnetic field $B$ perpendicular to the plane of the billiard is introduced by the "minimal substitution' for the canonical momentum

$$
\vec{p}=\vec{v}-\frac{1}{c} \vec{A}(\vec{r})
$$

where $\vec{v}=\vec{k}$ is the kinetic momentum and $\vec{A}(\vec{r})$ is the vector potential of the $B$ field. The classical paths $q$ consist now of segments of circular orbits with cyclotron radius $r_{c}=c k_{F} / B$. The corresponding action is

$$
S_{q}\left(k_{F}, B\right)=k_{F} L_{q}-\frac{1}{c} \int_{q} \vec{A} d \vec{r}
$$

Since the path $q$, which has the length $L_{q}$ and connects lead 1 with lead 2 is open, the classical action [Eq. (7)] and therefore the semiclassical Green's function [Eq. (5)] are not gauge invariant. However, in the presence of a magnetic field, the lead wave function [Eq. (3)] needs to be gauge transformed consistently with the gauge used for the cavity, even in the limit of weak fields. This introduces a phase distortion to the lead wave function. We will show below that incorporation of this distortion renders the semiclassical theory gauge invariant in the presence of a magnetic field.

For the calculation of the transmission coefficients, we construct asymptotic scattering states in the leads using the corresponding Landau gauges $(j=1,2)$,

$$
\vec{A}_{j}=B\left(-y_{j}, 0\right),
$$

when calculating the lead wave functions. ${ }^{21}$ We use a local coordinate system for each lead where $x_{j}$ denotes the direction along the lead and $y_{j}$ the transverse direction. Therefore $\vec{A}_{1}$ and $\vec{A}_{2}$ are different when the leads are not parallel. With these choices for the gauge, the $m$ th channel wave function can be written (like in the field free case) as a product of a plane wave in flux direction satisfying scattering boundary conditions for short-ranged potentials and a transverse wave function

$$
\psi_{m}\left(x_{j}, y_{j}\right)=e^{i k_{x_{j}} x_{j}} \phi_{m}\left(y_{j}\right)
$$

The transverse wave function satisfies

$$
\left[-\frac{1}{2} \frac{d^{2}}{d y_{j}^{2}}+V\left(y_{j}\right)+\frac{1}{2} \omega_{c}^{2}\left(y_{j}-y_{0}\right)^{2}\right] \phi_{m}\left(y_{j}\right)=E_{F} \phi_{m}\left(y_{j}\right)
$$

where $V\left(y_{j}\right)$ is the confining potential of the walls, $\omega_{c}$ $=B / c$ is the cyclotron frequency, and $y_{0}=c k_{x_{j}} / B$. This equation is usually solved numerically. ${ }^{23}$ In the following, we assume narrow leads and weak $B$ fields such that the cyclotron radius $r_{c}$ is large compared to the lead width $d$. In this limit, the diamagnetic term $\left(\propto \omega_{c}^{2}\right)$ can be neglected and the transverse wave functions are approximately given by the zero-field limit [Eq. (3)].

The crucial point to be noted is that $\phi_{m}\left(y_{1}\right)$ and $\phi_{n}\left(y_{2}\right)$ are constructed in different gauges, even though they do not explicitly depend on the magnetic field in the weak-field approximation. For the evaluation of the transmission amplitude in Eq. (4), they must be transformed to the reference gauge $\vec{A}$ in the interior of the cavity, yielding additional phase factors, $\exp \left[-(i / c) \Lambda_{j}\right]$, with 


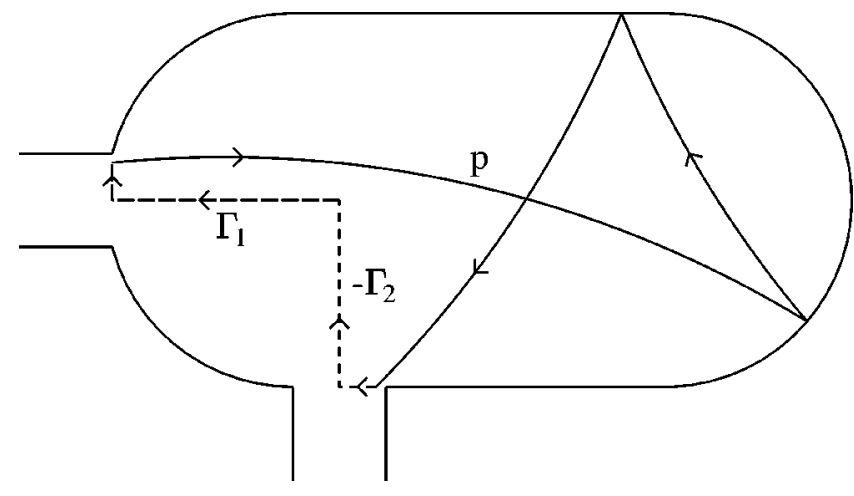

FIG. 1. Example for a real classical path $q$ (solid line) followed by virtual paths $\Gamma_{1}$ and $-\Gamma_{2}$ (dashed lines) generating the gaugeinvariant enclosed area of the trajectory.

$$
\Lambda_{j}(x, y)=\int_{\Gamma_{j}}\left(\vec{A}_{j}-\vec{A}\right) d \vec{r}
$$

where $\Gamma_{j}$ is a path starting at an arbitrary reference point $P_{0}$ and extending to the point $\left(x_{j}, y_{j}\right)$ where the path $q$ intersects the mouth of lead $j$. Inserting these additional phases into Eq. (4), the action $S_{q}$ in Eq. (5) must be replaced by

$$
\begin{aligned}
F_{q} & =S_{q}(B)+\frac{1}{c} \Lambda_{1}-\frac{1}{c} \Lambda_{2} \\
& =k_{F} L_{q}-\frac{1}{c}\left[\int_{q} \vec{A} d \vec{r}-\int_{\Gamma_{1}}\left(\vec{A}_{1}-\vec{A}\right) d \vec{r}+\int_{\Gamma_{2}}\left(\vec{A}_{2}-\vec{A}\right) d \vec{r}\right] \\
& =k_{F} L_{q}-\frac{1}{c}\left[\oint_{q-\Gamma_{2}+\Gamma_{1}} \vec{A} d \vec{r}-\int_{\Gamma_{1}} \vec{A}_{1} d \vec{r}+\int_{\Gamma_{2}} \vec{A}_{2} d \vec{r}\right] .
\end{aligned}
$$

Equation (12) is independent of the gauge $\vec{A}$ inside the cavity since the corresponding line integral is closed. A particularly useful choice of the path $\Gamma_{j}$ is shown in Fig. 1, where $P_{0}$ is at the intersection of the extension of the center of the leads into the cavity and $\Gamma_{j}$ goes from $P_{0}$ to the middle of the mouth of lead $j$ and from there to the point $\left(x_{j}, y_{j}\right)$. With this choice, the last two integrals on the right-hand side (RHS) of Eq. (12) are zero and by Stokes's theorem

$$
\begin{aligned}
F_{q} & =k_{F} L_{q}(B)-\frac{1}{c} \oint_{q-\Gamma_{2}+\Gamma_{1}} \vec{A} d \vec{r} \\
& =k_{F} L_{q}(B)-\frac{1}{c} B a_{q}(B),
\end{aligned}
$$

where $a_{q}$ denotes the directed gauge-invariant area.

The existence of a gauge-invariant phase has a simple geometric interpretation in terms of areas enclosed by closed loops. The loop consists of a combination of the classical path $q$ and the pseudopaths $-\Gamma_{2}$ and $\Gamma_{1}$. We call $\Gamma_{j}$ pseudopaths because the corresponding kinetic action for these segments is missing in Eq. (13). The contributions from pseudopaths were omitted in previous semiclassical analyses.

We thus arrive at the gauge-invariant expression of the semiclassical transmission amplitude:
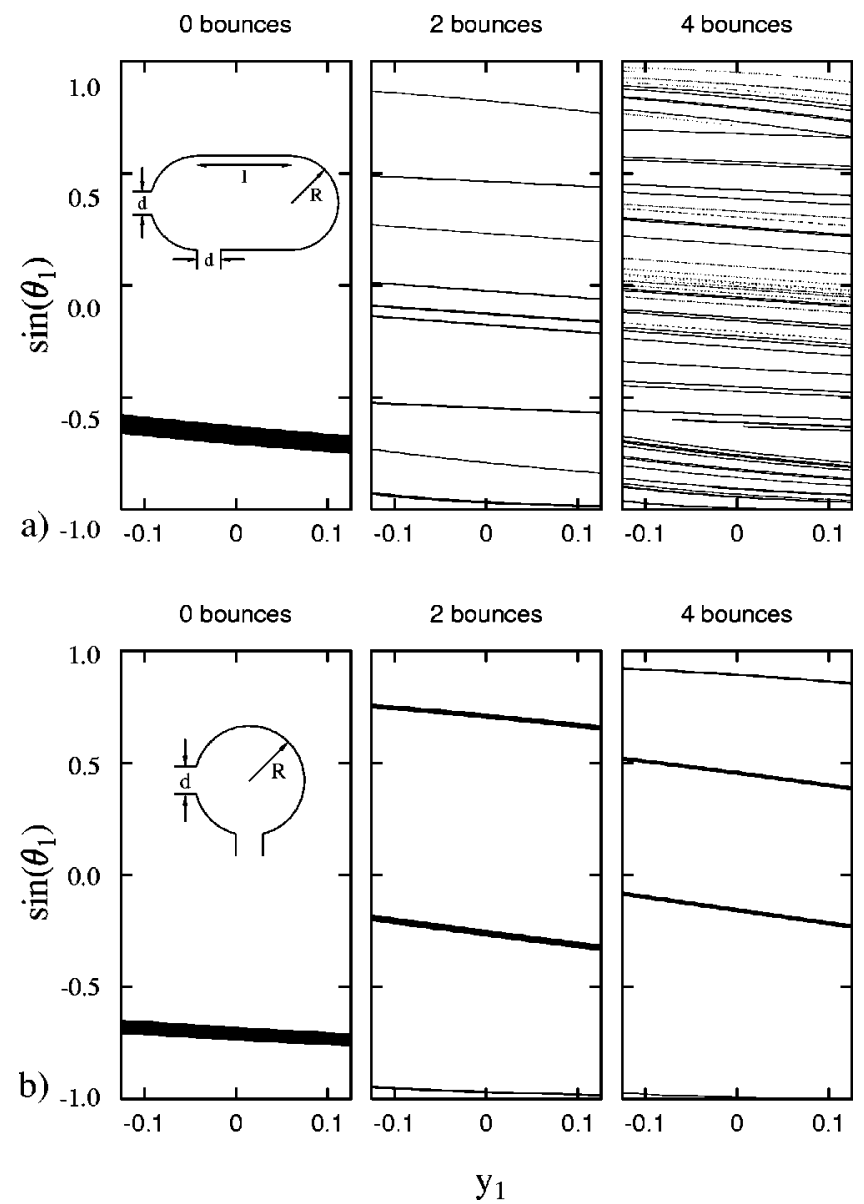

FIG. 2. Phase-space portraits for the transverse phase-space coordinates $\left(y_{1}, \sin \theta_{1}\right)$ at the entrance lead mouth of the billiard. Each connected area corresponds to one bundle reaching the exit lead after a specified number of bounces with the cavity walls: (a) circle with $R=\sqrt{4 / \pi+1}$ and $d=0.25$ and (b) stadium with $R=l / 2=1$ and $d=0.25$ (the parameters are chosen such that area $A$ of both cavities is the same). In the circle, the number of bundles grows linearly with the number of bounces whereas in the chaotic stadium, the number of bundles proliferates exponentially.

$$
\begin{aligned}
t_{m n}\left(k_{F}, B\right)= & -i \sqrt{v_{x, n} v_{x, m}} \frac{2 \pi}{(2 \pi i)^{3 / 2}} \sum_{\alpha} \int d y_{2} \int d y_{1} \\
& \times \phi_{n}^{*}\left(y_{2}\right) \phi_{m}\left(y_{1}\right)\left|D_{\alpha}\left(y_{1}, y_{2}, k_{F}, B\right)\right|^{1 / 2} \\
& \times \exp \left[i k_{F} L_{\alpha}\left(y_{1}, y_{2}, B\right)-\frac{i}{c} B a_{\alpha}\left(y_{1}, y_{2}, B\right)\right. \\
& \left.-i \frac{\pi}{2} \mu_{\alpha}\right] .
\end{aligned}
$$

The sum in Eq. (14) extends over all path bundles $\alpha$ rather than individual isolated paths. ${ }^{18}$ The underlying organization of transport in terms of bundles can easily be visualized by classical phase-space portraits. Figure 2 displays examples for the circle (an integrable system in the absence of the leads) and the Bunimovich stadium ${ }^{24}$ (its closed version is fully chaotic). For each trajectory specified by phase-space coordinates $\left(y_{1}, \sin \theta_{1}\right)$ at the entrance lead, we determine the domains of the constant number of bounces with the billiard walls before it reaches the exit lead. Each domain corre- 
sponds to a bundle of topologically equivalent trajectories. The transverse coordinate $y_{2}$ in the exit lead is a piecewise continuous function of the phase-space coordinates. The number and the size of the bundles are characteristically different for regular and chaotic structures. In the chaotic stadium the number of bundles proliferates exponentially with the number of bounces while the area in phase space $\left(\Delta y_{1} \Delta \sin \theta_{1}\right)$ occupied by each bundle decreases exponentially. With the increasing number of bounces, the mapping acquires a self-similar structure. Even at a number of only four bounces where the trajectories are still quite short ( $L$ $\$ 4 D$ ), already more than 50 bundles contribute to the transport through the stadium. In the circle, however, there are only four bundles at four bounces. This difference in the behavior of short paths is of practical importance since, because of the incoherent mean-free-path length in the experiments, long paths do not effectively contribute to transport. The dominant contribution comes from the few-bounce bundles, of which the occupied phase-space area is still relatively large.

The importance of the gauge invariance introduced here will be illustrated below for the directed area distributions that enter semiclassical estimates for weak localization and magnetoconductance autocorrelation functions $C(\Delta B)$.

\section{SEMICLASSICAL APPROXIMATIONS}

We discuss in this section a hierarchy of semiclassical approximations that result from additional approximations and simplifications of the fundamental semiclassical expression [Eq. (14)], some of which have already been discussed in the literature. ${ }^{10-19}$ We recall that Eq. (14) employs the semiclassical Green's function, or Fourier-Laplace transform of the Van Vleck propagator, ${ }^{22,9}$ for the motion in the interior of the billiard structure. No additional approximation has been made up to this point. In particular, the elements of the $S$ (or $T$ ) matrix are still evaluated as a projection of the Green's function onto the transverse (quantum-mechanical) eigenstates of the entrance or exit leads. Additional approximations are now invoked to perform the double integral in Eq. (14) pertaining to different limiting cases.

\section{A. Primitive semiclassical approximation}

In the primitive semiclassical (PSC) approximation, the remaining integrals over the lead mouths in Eq. (14) are performed in stationary-phase approximation (see, e.g., Ref. 12). The underlying assumption is that the phases in Eq. (14)

$$
F_{\alpha}\left(y_{1}, y_{2}, k_{F}, B\right)=k_{F} L_{\alpha}\left(y_{1}, y_{2}, B\right)-\frac{B}{c} a_{\alpha}\left(y_{1}, y_{2}, B\right),
$$

are rapidly varying over the integration interval $(-d / 2$ $\leqslant y_{1,2} \leqslant d / 2$ ). This, however, is only valid in the case when $k_{F} d \gg 2 \pi$, or equivalently in the high-mode limit $N \gg 1$. The transmission amplitude is then given by

$$
\begin{aligned}
t_{m n}^{(P S C)}\left(k_{F}, B\right)= & -\frac{(2 \pi i)^{1 / 2}}{2 d} \sum_{q(\bar{n}, \bar{m})} \operatorname{sgn}(\bar{n}) \operatorname{sgn}(\bar{m})\left|\widetilde{D}_{q}\right|^{1 / 2} \\
& \times \exp \left[i \widetilde{F}_{q}\left(k_{F}, B\right)-i \frac{\pi}{2} \tilde{\mu}_{q}\right]
\end{aligned}
$$

where the sum extends over all isolated paths, with $\bar{n}=$ $\pm n, \bar{m}= \pm m$, for which the transverse momenta $k_{y_{1}}$ and $k_{y_{2}}$ are conserved at the entrance and exit leads, i.e.,

$$
\begin{aligned}
& k_{y_{1}}=k_{F} \sin \theta_{1}= \pm m \pi / d, \\
& k_{y_{2}}=k_{F} \sin \theta_{2}= \pm n \pi / d .
\end{aligned}
$$

The weighting factor for each path is

$$
\widetilde{D}_{q}=\left.\frac{1}{k_{F}} \frac{\partial y_{1}}{\partial\left(\sin \theta_{2}\right)}\right|_{\sin \theta_{1}},
$$

$\theta_{1}$ and $\theta_{2}$ are the incoming and outgoing angles of the path, and $\tilde{\mu}_{q}$ is the modified Maslov index due to the additional singularities of $\widetilde{D}_{q}$. The interference phase is determined by the compensated action of each path

$$
\widetilde{F}_{q}\left(k_{F}, B\right)=F_{q}+k_{y_{1}} y_{1}+k_{y_{2}} y_{2} .
$$

In previous studies (e.g., Ref. 12), Eq. (16) was evaluated in the limit of a weak magnetic field. In this limit, the B field is included through the Aharonov-Bohm phase $B a_{q} / c$ while the classical path is taken as a straight-line trajectory. This accounts for magnetic-field effects to first order, i.e., orbital paramagnetism. ${ }^{27}$ Terms of higher order, i.e., diamagnetic corrections, which are of the order $B^{2}$ and enter through the curvature of the classical paths, are neglected. For a chaotic billiard, such as the stadium, however, the validity of this approximation is not obvious, since a chaotic system does not only display exponential sensitivity to initial conditions but also exponential sensitivity to perturbations. Figure 3 shows the phase-space portrait for two selected bounce numbers at $B=0$ (straight trajectories) and at a magnetic field that corresponds to a cyclotron radius $r_{c}=25 R$, i.e., about one order of magnitude larger than the linear dimension of the stadium. It illustrates that, even in a strongly chaotic cavity, the dominant bundles in the phase space are structurally stable. This means that, in spite of the sensitivity to perturbations, for small variations $\Delta B$, it is possible to vary $y_{1}$ and $y_{2}$ for $B+\Delta B$ such that the resulting path has the same bouncing pattern and the same initial and final angle as the corresponding path at $B$. A Taylor expansion for the compensated action [Eq. (19)] yields

$$
\begin{aligned}
\widetilde{F}_{q}(B+ & \Delta B) \\
= & \widetilde{F}_{q}(B)-\frac{a_{q}}{c} \Delta B \\
& +\left(k_{F} \frac{\partial\left(L_{q}+\sin \theta_{1} y_{1}+\sin \theta_{2} y_{2}\right)}{\partial B}-\frac{B}{c} \frac{\partial a_{q}}{\partial B}\right) \Delta B \\
& +O\left(\Delta B^{2}\right) .
\end{aligned}
$$

A numerical evaluation of the two derivative terms in the parentheses shows that they cancel out within each bundle, provided that $\sin \theta_{1}$ and $\sin \theta_{2}$ are kept constant and provided that the gauge-invariant area is used. It should be noted that this cancellation is not trivial. If one either uses open (nongauge-invariant) paths or mixed boundary conditions (e.g., $y_{1}$ and $\sin \theta_{1}$ fixed), curvature-dependent linear corrections in $\Delta B$ are nonvanishing. The origin of the cancellation lies in the fact that each trajectory consists of a set of circular seg- 

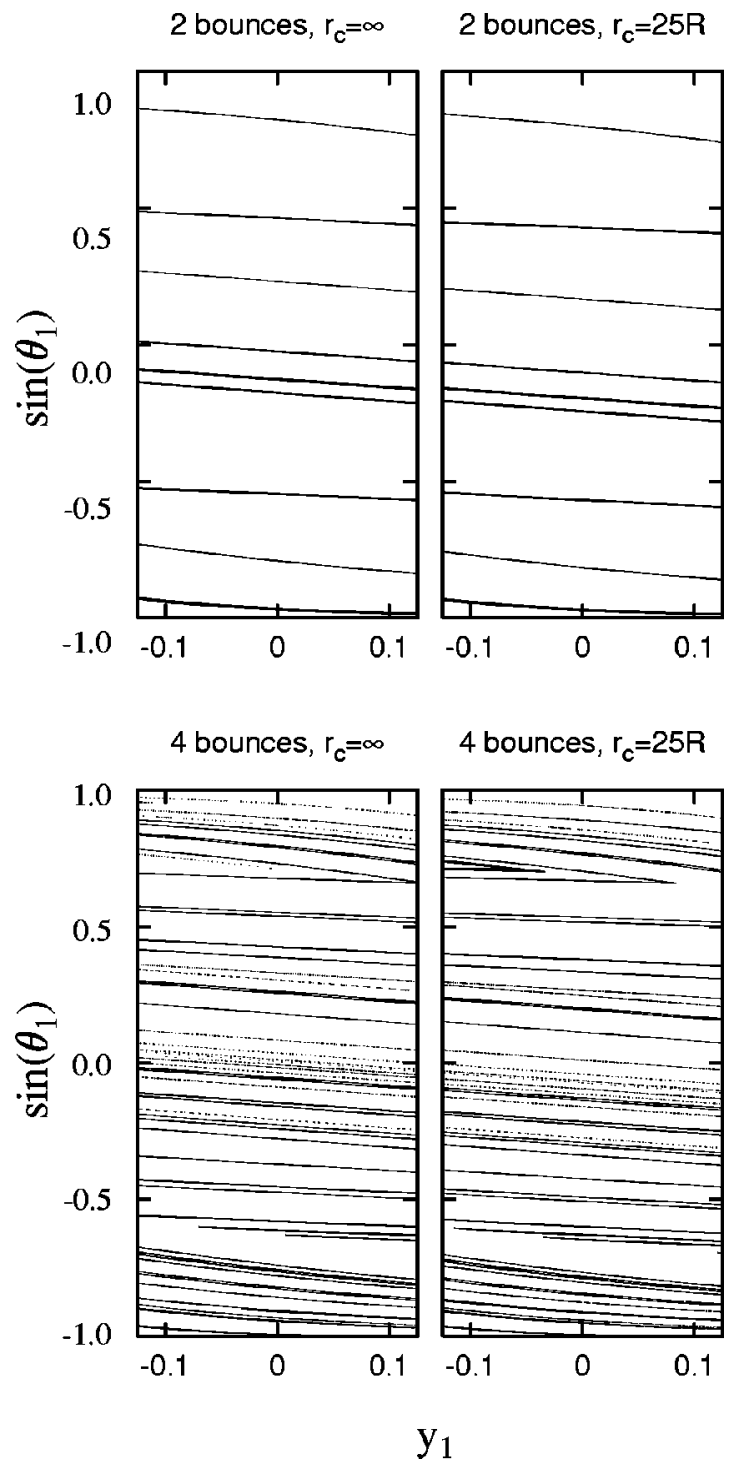

FIG. 3. Phase-space map for the bundles with two and four bounces in the chaotic stadium [inset of Fig. 2 (a)] for two different magnetic-field strengths corresponding to a cyclotron radius $r_{c}$ $=\infty$ and $r_{c}=25 R \approx 10 \sqrt{A}$. The bundles are structurally stable when the magnetic field is varied as long as $r_{c} \gg \sqrt{A}$.

ments. For a closed circle, the relation $r d L / d r-d a / d r=0$ holds, where $r$ is the radius, $L$ is the circumference, and $a$ is the area. For a sequence of segments each followed by a specular reflection at the wall, the cancellation still holds provided homogeneous boundary conditions $\left(\sin \theta_{1}\right.$ and $\sin \theta_{2}$ fixed or $y_{1}$ and $y_{2}$ fixed) are imposed on the trajectory.

The Taylor expansion of the action within the bundle reduces then to

$$
\widetilde{F}_{q}(B+\Delta B)=\widetilde{F}_{q}(B)-\frac{a_{q}}{c} \Delta B+O\left(\Delta B^{2}\right),
$$

i.e., to first order, the variation $\Delta B$ only appears as a change in the Aharonov-Bohm phase, leaving the path unchanged. This justifies the use of straight-line trajectories for weak magnetic fields. Equation (21) is important for the semiclas- sical expressions of magnetoconductance fluctuations, where paths from the same bundle but at different magnetic fields enter (see below).

\section{B. Fraunhofer diffraction approximation}

The stationary-phase approximation for the evaluation of the integrals over the lead mouths in Eq. (14) is invalid for low-mode numbers, i.e., small $k_{F}$. The key observation is that within each bundle (Fig. 2), the classical action $F_{\alpha}\left(y_{1}, y_{2}, k_{F}, B\right)$ [Eq. (13)] varies only slowly. The variation is of the order of $k_{F} d$. Since $k_{F}$ is of the order of $N \pi / d$, the variation of the action is $\Delta F_{q} \lesssim N \pi$. Therefore, unless the number of open channels $N$ is large, the evaluation of the double integral in Eq. (14) by stationary-phase approximation, which requires $\Delta F_{q} \gg 2 \pi$, is bound to fail. The primitive semiclassical (PSC) approximation can only be expected to work well in the case of high-mode numbers, if at all. Moreover, for direct collision-free trajectories as well as for bundles that only collide with straight sections of the microstructure, the PSC approximation breaks down since trajectories satisfying the transverse momentum conservation [Eq. (17)] are not isolated but form continuous manifolds.

We therefore implement a modification of the semiclassical treatment of the transmission amplitude, which holds for low-mode numbers and all geometries, by using a more accurate approximation for the double integral over all path bundles. We rewrite Eq. (14) as

$$
\begin{aligned}
t_{m n}^{(S C)}= & -\sqrt{v_{x, m} v_{x, n}} \frac{2 \pi i}{(2 \pi i)^{3 / 2}} \\
& \times \sum_{\alpha} \exp \left[i \bar{F}_{\alpha}\left(k_{F}, B\right)-i \frac{\pi}{2} \mu_{\alpha}\right] H_{\alpha}\left(m, n, k_{F}\right),
\end{aligned}
$$

where $\bar{F}_{\alpha}$ is the average classical compensated action for each bundle, evaluated at $y_{1}=y_{2}=0$. The amplitude factor for each bundle $H_{\alpha}$ is

$$
\begin{aligned}
H_{\alpha}\left(m, n, k_{F}\right)= & \int d y_{2} \int d y_{1} \phi_{n}^{*}\left(y_{2}\right) \phi_{m}\left(y_{1}\right) \\
& \times\left|D_{\alpha}\left(y_{1}, y_{2}, k_{F}\right)\right|^{1 / 2} \exp \left[i \left\{F_{\alpha}\left(y_{1}, y_{2}, k_{F}, B\right)\right.\right. \\
& \left.\left.-\bar{F}_{\alpha}\left(k_{F}, B\right)\right\}\right] .
\end{aligned}
$$

The integrand in the amplitude factor $H_{\alpha}$ is, in general, a weakly varying function of $y_{1}$ and $y_{2}$ for small $m$ and $n$. Only in the limit of a large number of open channels $\left(k_{F}\right.$ $\rightarrow \infty$ ), it displays rapid oscillations by which the stationaryphase limit [Eq. (16)] is recovered. In Eq. (23) the coupling to the lead is treated quantum mechanically. Physically, the latter implies the nonconservation of transverse momentum [Eq. (17)] at the entrance and exit leads. The integral over the transverse coordinates in Eq. (23) for fixed-channel numbers $m$ and $n$ amounts to an integration over nonclassical paths connecting the entrance and the exit leads with the interior of the cavity. In this way Eq. (23) automatically takes into account diffraction of the wave at the entrance and exit leads. 
The transmission amplitude $t^{(S C)}$ [Eq. (22)] can be evaluated analytically to a good approximation. ${ }^{18}$ Since $D_{\alpha}\left(y_{1}, y_{2}, k_{F}, B\right)$ varies very smoothly within a given bundle $\alpha$, we replace it by its value at the center of bundle $\bar{D}_{\alpha}\left(k_{F}, B\right)$. For the exponent in Eq. (23), we use a Taylor expansion to first order in $y_{1}$ and $y_{2}$ :

$$
F_{\alpha}\left(y_{1}, y_{2}, k_{F}, B\right)-\bar{F}_{\alpha}\left(k_{F}, B\right) \approx-k_{F} \sin \theta_{1} y_{1}+k_{F} \sin \theta_{2} y_{2} .
$$

Keeping only terms up to first order corresponds to a Fraunhofer diffraction approximation (FDA) to the diffraction integral [Eq. (23)]. The FDA transmission amplitude is given by

$$
\begin{aligned}
t_{m n}^{(F D A)}\left(k_{F_{1}} B\right)= & -\frac{1}{(2 \pi i)^{1 / 2}} \frac{2}{d} \sqrt{k_{x, n} k_{x, m}} \sum_{\alpha}\left|\bar{D}_{\alpha}\left(k_{F}, B\right)\right|^{1 / 2} \\
& \times h_{m}^{*}\left(k_{F}, \sin \theta_{1}\right) h_{n}\left(k_{F}, \sin \theta_{2}\right) \\
& \times \exp \left[i\left(k_{F} \bar{L}_{\alpha}-\frac{1}{c} B \bar{a}_{\alpha}\right)-i \frac{\pi}{2} \mu_{\alpha}\right]
\end{aligned}
$$

with

$$
\begin{aligned}
h_{n}\left(k_{F}, \sin \theta\right)= & i^{n-1} \frac{\sin \left[\left(\frac{n \pi}{d}+k_{F} \sin \theta\right) \frac{d}{2}\right]}{\frac{n \pi}{d}+k_{F} \sin \theta} \\
& -(-i)^{n+1} \frac{\sin \left[\left(\frac{n \pi}{d}-k_{F} \sin \theta\right) \frac{d}{2}\right]}{\frac{n \pi}{d}-k_{F} \sin \theta}
\end{aligned}
$$

and

$$
\bar{D}_{\alpha}\left(k_{F}, B\right)=\frac{1}{k_{F} \cos \theta_{1} \cos \theta_{2}}\left|\frac{\partial \sin \theta_{1}}{\partial y_{2}}\right|_{y_{1}=y_{2}=0} .
$$

Alternative diffraction approximations using Kirchhoff's diffraction theory have been introduced in Ref. 17.

\section{CTMC method}

One important feature of the Fraunhofer diffraction approximation is the incorporation of the bundle structure and of diffraction effects, i.e., the breaking of the correlation between launching angle and the transverse-mode quantum number. These features can be preserved in simplified form with the help of the classical trajectory Monte Carlo (CTMC) method. ${ }^{25,26}$ In the CTMC method, the PSC approximation is assumed to be valid for the evaluation of the classical quantities $\widetilde{D}_{q}$ and $\widetilde{F}_{q}$. However, the mapping of quantum numbers in the quantum wires onto the initial and final conditions for classical trajectories inside the cavity employs phasespace binning. Accordingly, each quantum number is associated with a classical bin within each of which a microcanonical distribution of trajectories is assumed (alternative distributions are possible). These techniques have found widespread applications in the theory of ion-atom collisions and chemical physics (see, e.g., Refs. 25 and 26). In the present case, the CTMC method maps the quantum numbers in the wires onto the classical angular distribution inside the cavity in terms of bins of the classical phase space in the transverse degree of freedom according to the microcanonical ensemble. Each quantum number $m$ is mapped onto the uniform distribution of launching angles corresponding to a uniform distribution of transverse momentum, or equivalently, of $\sin \theta_{1}$ within the bin

$$
\left|k_{F} \sin \theta_{1} \pm \frac{m \pi}{d}\right| \leqslant \frac{\pi}{d}
$$

Furthermore, for a constant potential across the mouth of the lead, also the microcanonical position distribution is uniform in $y_{1}$. An equivalent binning is performed for the arrival angle $\theta_{2}$ in order to map the classical angular distribution onto the quantum number of the exit lead. Consequently, each mode number for a given $k_{F}$ is associated with a distribution of launching (and arrival) angles [Eq. (28)] rather than the discrete values of Eq. (17).

Binning techniques can be understood as a way to introduce nonclassical paths, more precisely, paths associated with nonclassical initial conditions. Nonconservation of the transverse momentum is introduced within the width of the bin. The CTMC method allows us therefore to partially treat diffraction effects where the width of zero-order diffraction maxima is well represented while the higher-order diffraction peaks are missing. ${ }^{19}$

\section{Classical limit}

The classical limit of the semiclassical approximation can be extracted from the PSC expression [Eq. (16)] by decomposing the transmission (or reflection) coefficient $T_{m n}^{(P S C)}$ $=\left|t_{m n}^{(P S C)}\right|^{2}$ into a diagonal and off-diagonal component with respect to the path index $q$. The "classical" part $T_{m n}^{c l}$ $=\Sigma_{q=q^{\prime}}($ ) excludes interferences between different paths while the nonclassical part $T_{m n}-T_{m n}^{c l}=\Sigma_{q \neq q^{\prime}}$ () contains the quantum interferences. Following an argument by Baranger et al., ${ }^{12,15}$ the diagonal "classical" part can be shown to yield the classical transmission probability $T_{m}$, i.e., the probability, that a trajectory with an entrance angle $\theta_{1}$ corresponding to mode $m$ but arbitrary transverse coordinate $y_{1}$, leaves the stadium via the exit lead

$$
T_{m}^{c l}=\sum_{n} T_{m n}^{c l}=\frac{\pi}{2 d^{2}} \sum_{n} \sum_{q}\left|\widetilde{D}_{q}\right|
$$

For high-mode numbers, the sum over $n$ can be converted into an integral over the exit angle $\theta_{2}$ :

$$
\sum_{n} \rightarrow \frac{d k_{F}}{\pi} \int d\left(\sin \theta_{2}\right)
$$

The sum extends now over all bundles $\beta$, which, in this context, consist of sets of paths that have the same entrance angle, corresponding to mode $m$, but a variable exit angle, Within each bundle, the integral extends from the minimum to the maximum exit angle, 


$$
\sum_{n} T_{m n}^{c l}=\frac{d k_{F}}{\pi} \sum_{\beta} \int_{\left(\sin \theta_{2}\right)_{\min }}^{\left(\sin \theta_{2}\right)_{\max }} d\left(\sin \theta_{2}\right)\left|\widetilde{D}_{\beta}\right|
$$

Inserting $\left|\widetilde{D}_{\beta}\right|=\left|\left(1 / k_{F}\right)\left(\partial y_{1} / \partial \sin \theta_{2}\right)\right|_{\sin \theta_{1}}$ yields

$$
\sum_{n} T_{m n}^{c l}=\frac{1}{2 d} \sum_{\beta} \int_{\left(y_{1}\right)_{\min }}^{\left(y_{1}\right)_{\max }} d y_{1} .
$$

This is just the total classical transmission probability for trajectories entering the cavity with an angle belonging to mode number $m$. The same argument holds for the sum over reflected trajectories and the classical reflection probability $R_{m}^{c l}$. Since an electron will leave the cavity either through the exit or the entrance lead,

$$
T_{m}^{c l}+R_{m}^{c l}=1
$$

Equation (33) can be understood as a classical unitarity (or flux conservation) relation. As will be discussed below, it is the off-diagonal contributions from quantum interferences that lead to violation of unitarity in the semiclassical approximation.

\section{MAGNETOCONDUCTANCE FLUCTUATIONS}

Magnetotransport displays strongly irregular yet reproducible fluctuations as a function of both the effective Fermi wave number $k_{F}$ and the magnetic field $B$. Most experimental studies are performed as a function of $B$, which is easier to manipulate than the Fermi energy of the structure. Magnetoconductance fluctuations in ballistic quantum transport are far from being random but possess long-range correlations. The goal of semiclassical analysis is to relate the magnetoconductance fluctuations to the underlying classical dynamics of ballistic transport.

\section{A. Area spectrum}

On the most elementary level, this can be done by studying the power spectrum of the transmission amplitude for a fixed pair of mode numbers

$$
\left|\tilde{t}_{m n}\left(k_{F}, a\right)\right|^{2}=\left|\frac{1}{B_{\text {max }}} \int_{0}^{B_{\text {max }}} d B e^{i a B / c} t_{m n}\left(k_{F}, B\right)\right|^{2} .
$$

Accordingly, the total power spectrum is given by

$$
P_{t o t}\left(k_{F}, a\right)=\sum_{m, n}\left|\tilde{t}_{m n}\left(k_{F}, a\right)\right|^{2} .
$$

The variable conjugate to $B$ is the effective area $a$ within the framework of semiclassical approximation. The power spectrum at fixed $k_{F}$ can be identified with the spectrum of areas enclosed by classical trajectories. Similar to the case of the length spectrum for the field free transmission, ${ }^{16-18,28}$ the choice of the integration interval of the Fourier integral $\left[0, B_{\text {max }}\right]$ has nontrivial consequences beyond the limitation of the resolution. Since the curvature of the paths and, hence, the enclosed areas change as functions of the magnetic field, the resulting area spectrum is to be understood as an average over area distributions pertaining to different fields.

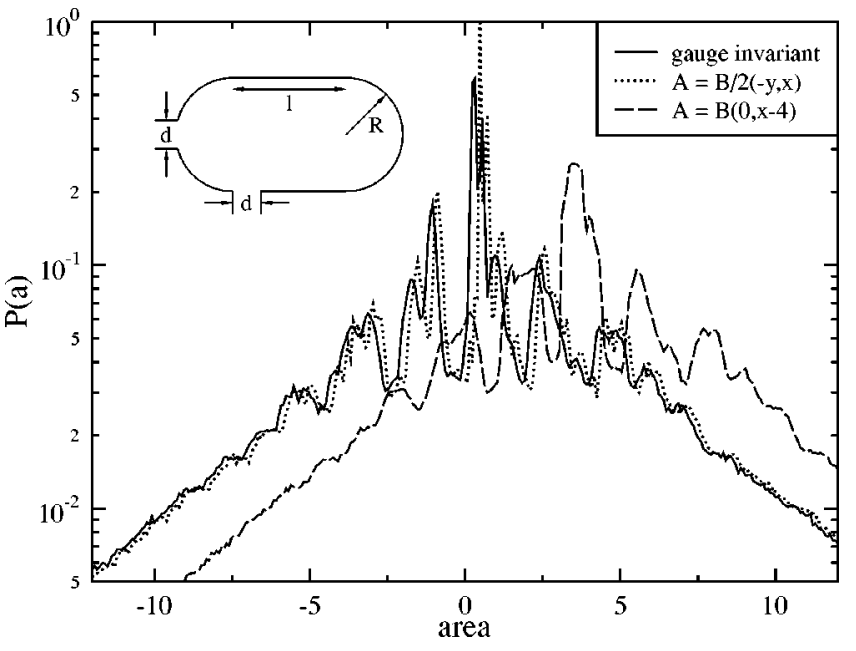

FIG. 4. Directed area distribution function $P(a)$ in gaugeinvariant $\left(a=\oint_{q-\Gamma_{2}+\Gamma_{1}} \vec{A} d \vec{r}\right)$ and non-gauge-invariant ( $a$ $=\int_{q} \vec{A} d \vec{r}$ ) form with different gauges for the stadium billiard with $R=l / 2=1$ and $d=0.25$. The exponential decay of larger areas is a universal feature of chaotic structures and the slope is gauge independent, but the nonuniversal behavior of the area distribution at small values strongly depends on the gauge.

We focus first on the classical area spectrum $P_{c l}(a)$ at fixed magnetic field. $P_{c l}(a)$ corresponds to the area spectrum within the framework of the CTMC simulation. Calculations have been performed for the integrable circle billiard and the chaotic stadium billiard.

Figure 4 displays the directed area distribution $P(a)$ calculated in both non-gauge-invariant and gauge-invariant form for the $90^{\circ}$ stadium geometry. A large number of initial trajectories $\left(\geqslant 10^{6}\right)$ are launched with initial conditions at the entrance lead uniformly distributed in phase space, i.e., uniform in $y_{1}$ and in $\sin \theta_{1}$, and for each trajectory the enclosed directed area is recorded. Several features are worth noting. For larger enclosed areas, we find an approximately exponential "universal" distribution in agreement with previous investigations. ${ }^{12,13}$ Moreover, the exponential decay constant is reproduced even if the non-gauge-invariant form is used. For small and intermediate values of the area $a$, however, the distribution functions are highly structured functions and strongly gauge dependent. These nonexponential structures, missing in previous studies (see, e.g., Ref. 12), contain the geometry-specific information of ballistic transport and of magnetoconductance fluctuations. We therefore arrive at the conclusion that the calculation of the semiclassical magnetoconductance correlation function (see below) beyond the simple "universal" exponential distribution requires a gauge-invariant description. All Aharonov-Bohm phases and resulting area distributions discussed in the following are calculated in gauge-invariant form.

The magnetic-field dependence of the area distribution is displayed in Fig. 5. As anticipated from the structural stability of bundles under variation of the $B$ field (see Fig. 3), the $B$-field dependence of $P(a)$ is weak as long as the cyclotron radius $r_{c}$ remains about an order of magnitude larger than the linear dimension of the cavity, $r_{c}>10 \sqrt{A}$. Within this limitation, the Fourier transform [Eq. (34)] of the quantum and 


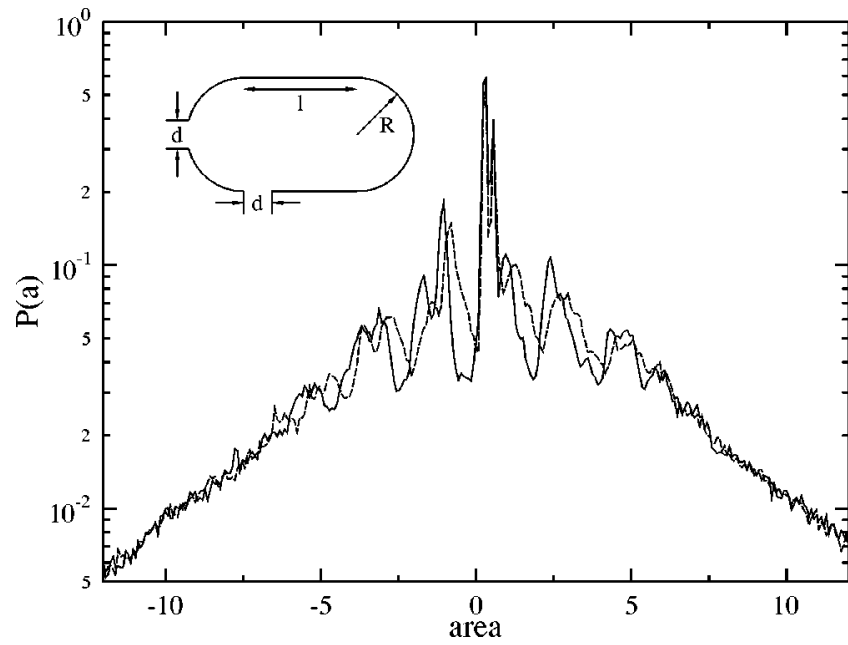

FIG. 5. Dependence of the classical area distribution $P_{c l}(a)$ on the magnetic field (cyclotron radius): $r_{c}=\infty$ (solid line), $r_{c}$ $=10 \sqrt{A}$ (dashed line). Because of the structural stability of bundles under a change of magnetic field (see Fig. 3), the principal features of the nonuniversal distribution of small areas are the same in both curves and the universal exponential decay at larger areas is not affected.

semiclassical amplitude can be associated with a single (broadened) area distribution.

Comparison between the classical area distribution $P_{c l}$ and the area spectrum within the PSC approximation is shown in Fig. 6 for the circular billiard with the two leads at an angle of $90^{\circ}$ to each other. Note that for an infinite integration interval over $B$ but the underlying classical dynamics kept at fixed $B$, Eq. (34) implies that the two should agree provided that the sum over all paths has converged. The observed level of agreement is therefore primarily a measure for the completeness of the sum over all paths in Eq. (16). In the present example, about 18000 trajectories are included. It should be noted that $P(a)$ is not symmetric with respect to the reversal of the sign of the directed area $(a \rightarrow-a)$, or equivalently, the transmission amplitude is not invariant un-

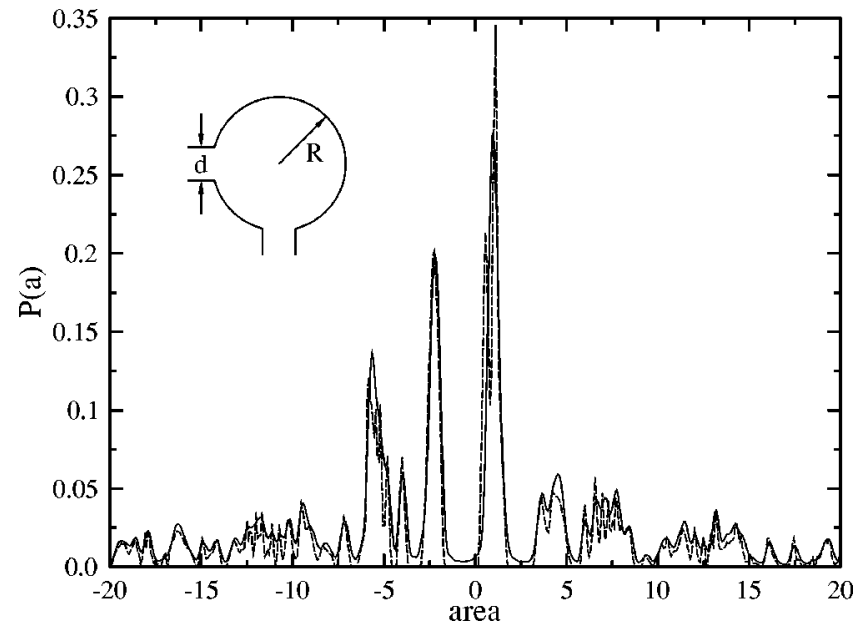

FIG. 6. Comparison between the total power spectrum $\sum_{m, n}\left|\widetilde{t}_{m n}^{(P S C)}(a)\right|^{2}$ at $k_{F}=50.5 \pi / d$ and the classical area distribution $P_{c l}(a)$ for a circle with perpendicular leads $(R=\sqrt{4 / \pi+1}, d$ $=0.4)$. der the inversion of the field $(B \rightarrow-B)$ since the $90^{\circ}$ geometry of the attached quantum wires breaks the reflection symmetry of the scattering problem.

One important difference to previously investigated length spectra ${ }^{16-18}$ (power spectra of $t_{m n}$ as a function of $k$ ) is that the association of pronounced peaks with individual bundles of trajectories is not clear cut. One reason for this is that the directed area, unlike the length, is not positive definite. Therefore, long trajectories can have small enclosed areas, just as short trajectories, because of strong cancellation effects of directed areas segments of opposite sign. This cancellation effect results in the accumulation of trajectories with small directed areas.

\section{B. Conductance fluctuations and autocorrelation functions}

Experimentally accessible is the magnetoconductance as a function of the applied magnetic field

$$
T_{m n}(B)=\left|t_{m n}(B)\right|^{2} .
$$

Within the semiclassical approximation, $T_{m n}(B)$ depends on pairs of trajectories (or bundles) and, correspondingly, differences between directed areas. In the PSC approximation we have

$$
\begin{aligned}
T_{m n}^{(P S C)}(B)= & \frac{\pi}{2 d^{2}} \sum_{q, q^{\prime}} \operatorname{sgn}(\bar{m}) \operatorname{sgn}(\bar{n}) \operatorname{sgn}\left(\bar{m}^{\prime}\right) \operatorname{sgn}\left(\bar{n}^{\prime}\right) \\
& \times\left|\widetilde{D}_{q} \widetilde{D}_{q^{\prime}}\right|^{1 / 2} \exp \left[i\left[\widetilde{F}_{q}(B)-\widetilde{F}_{q^{\prime}}(B)\right]\right. \\
& \left.-i \frac{\pi}{2}\left(\tilde{\mu}_{q}-\tilde{\mu}_{q^{\prime}}\right)\right]
\end{aligned}
$$

and, correspondingly, in the FDA

$$
\begin{aligned}
T_{m n}^{(F D A)}(B)= & \frac{v_{x, m} v_{x, n}}{2 \pi} \sum_{\alpha, \alpha^{\prime}} H_{\alpha}\left(m, n, k_{F}\right) H_{\alpha^{\prime}}^{*}\left(m, n, k_{F}\right) \\
& \times \exp \left[i\left[\bar{F}_{\alpha}(B)-\bar{F}_{\alpha^{\prime}}(B)\right]-i \frac{\pi}{2}\left(\mu_{\alpha}-\mu_{\alpha^{\prime}}\right)\right] .
\end{aligned}
$$

In both approximations [Eqs. (37) and (38)], the oscillatory component is determined by differences between directed areas. The cancellation effects and hence the difficulty in mapping Fourier components onto distinct classical bundles becomes even more severe than for the power spectrum of the transmission amplitude. Cancellation effects now take place not only between different segments of a given trajectory but also between directed areas of different trajectories resulting in pronounced accumulation of Fourier components with $a \approx 0$ in the power spectrum of $T$.

The magnetoconductance calculated in the PSC approximation using Eq. (37) should agree with the CTMC result provided the off-diagonal terms in the path index are neglected. In this case, the agreement provides an indication for the completeness of the path sum. An example is shown in Fig. 7 for the circle with leads at an angle of $90^{\circ}$ relative to each other. The overall agreement is very good. The most pronounced deviation occurs at very low $m$ (classical shoot- 


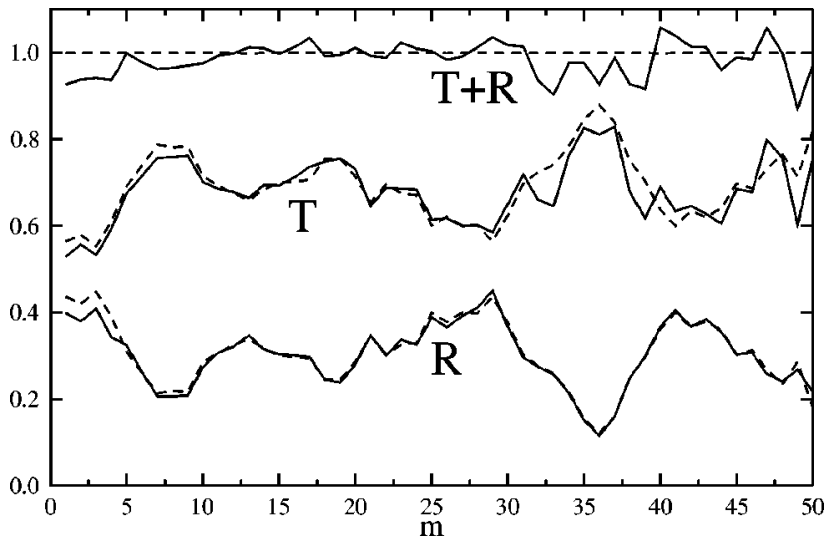

FIG. 7. Classical transmission $\left(T_{m}=\Sigma_{n} T_{m n}\right)$ and reflection $\left(R_{m}=\Sigma_{n} R_{m n}\right)$ in the circle billiard (inset of Fig. 6) as a function of entrance lead mode number $m$ at $k_{F}=50.5 \pi / d$, i.e., 50 open modes. Dashed lines, CTMC simulation; solid lines, result of Eq. (29).

ing angle near $\theta \simeq 0$ ) and at high $m$. The failure near $\theta=0$ results from the fact that there is no $m=0$ state, which would accommodate classical trajectories with $\theta=0 .{ }^{15}$ The deviation at high $m$ results from the fact that for the corresponding shooting angles most trajectories belong to the direct bundle for which the PSC approximation fails and which is therefore calculated by direct numerical evaluation of the double integral in Eq. (14).

Fluctuations in the conductance can be conveniently described by the autocorrelation function

$$
C(\Delta B)=\langle\delta T(B+\Delta B) \delta T(B)\rangle,
$$

where $\delta T(B)=T(B)-\langle T(B)\rangle$ and the ensemble average is taken either over a range of $k_{F}$ or a range of $B$ fields. With the help of Eqs. (37) and (38), $C(\Delta B)$ can be evaluated in the semiclassical, as well as primitive semiclassical, approximations. The value of the non-normalized correlation function at zero field $C(\Delta B=0)$ gives the mean-squared conductance fluctuation $\left\langle\delta T^{2}\right\rangle$, which has been extensively investigated within the framework of random matrix theory. ${ }^{29}$ Frequently, the correlation function $C(\Delta B)$ is normalized such that $C(0)=1$. The correlation function $C(\Delta B)$ is customarily evaluated within the so-called "diagonal approximation", 12

$$
\begin{aligned}
C(\Delta B) & \approx \sum_{n, m} C_{m n}(\Delta B) \\
& =\sum_{n, m}\left\langle\delta T_{m n}(B+\Delta B) \delta T_{m n}(B)\right\rangle_{B} .
\end{aligned}
$$

Here, correlations between fluctuations in different modes are neglected. Using the PSC amplitudes [Eq. (37)] and the Taylor expansion [Eq. (21)], the correlation function is reduced to

$$
C_{m n}(\Delta B)=\left(\frac{\pi}{2 d^{2}}\right)^{2} \sum_{q \neq q^{\prime}}\left|\widetilde{D}_{q} \widetilde{D}_{q^{\prime}}\right| \exp \left[\frac{i}{c} \Delta B\left(a_{q}-a_{q^{\prime}}\right)\right] .
$$

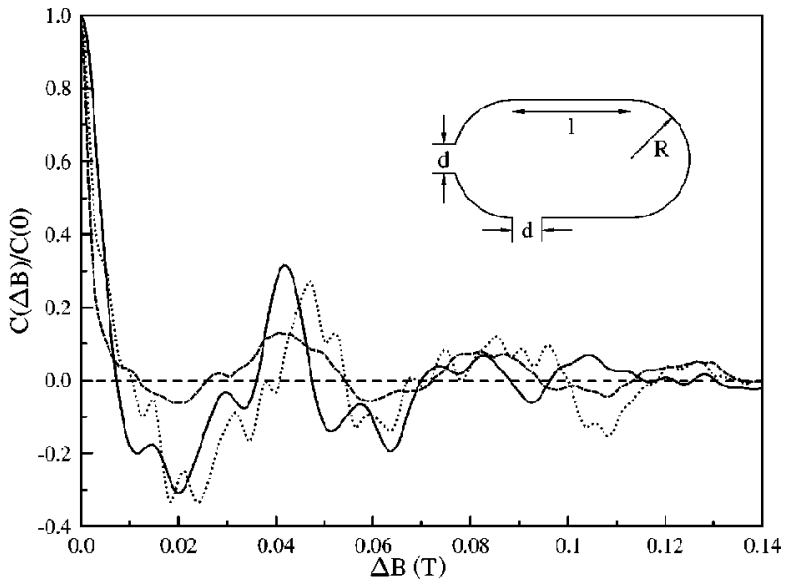

FIG. 8. Comparison of magnetoconductance autocorrelation functions obtained with the CTMC binning method (dashed curve), FDA for $k_{F}=3.5 \pi / d$ (solid curve), and the experimental data (Ref. 19 , dotted curve) in the stadium with $l / 2=R=1$ and $d=0.4$. For unit conversion we used an effective area in the experiment of $A_{\text {eff }}=0.5 \mu \mathrm{m}$.

The sum over bundles in Eq. (41) can be transformed into an integral over enclosed area differences between pairs of paths, ${ }^{19}$

$$
C_{m n}(\Delta B)=\int_{-\infty}^{\infty} d a P_{2}^{m, n}\left(a=a_{q}-\left.a_{q^{\prime}}\right|_{q \neq q^{\prime}}\right) \exp \left[-\frac{i}{c} \Delta B a\right] .
$$

$P_{2}^{m, n}$ denotes the correlation function for the area difference between pairs of paths, $q$ and $q^{\prime}$, having a difference in directed area of $a=a_{q}-a_{q^{\prime}}$ with $\sin \theta_{q}$ and $\sin \theta_{q^{\prime}}$ lying in the bin of the entrance angle $m$ and of the exit angle $n$ as defined in Eq. (28).

We notice that the binning technique [Eq. (28)] is of crucial importance for uncovering oscillatory structures in $C(\Delta B) . P_{2}^{m, n}$ contains the information on the angular correlation between bundles connecting a given set of modes $(m, n)$. Without binning, only a positive-definite correlation function of Lorentzian form can be obtained as in previous studies. ${ }^{12,13}$ This is demonstrated in the following way: Without binning, the pair distribution function $P_{2}(a)$ can be expressed by the self-convolution of the simple area distribution $P(a)$,

$$
P_{2}(a)=\int_{-\infty}^{\infty} d a^{\prime} P\left(a^{\prime}+a\right) P(a) .
$$

The correlation function $C(\Delta B)$ is then reduced to a square of the Fourier transform of the directed area distribution

$$
C(\Delta B)=\frac{1}{4}\left|\int d a P(a) \exp \left[-\frac{i}{c} \Delta B a\right]\right|^{2},
$$

which is positive definite. If we further assume a "universal" exponential distribution for the area, $P(a) \propto \exp [-\beta a]$, the autocorrelation reduces to a squared Lorentzian ${ }^{12,13}$

$$
C(\Delta B)=C(0) /\left[1+(1 / c)^{2}(\Delta B / \beta)^{2}\right]^{2},
$$

a frequently invoked simplified model that has been used to fit the behavior of $C(\Delta B)$ for small magnetic fields $\Delta B .^{2-4}$

In Figs. 8 and 9 we present the comparison between ex- 


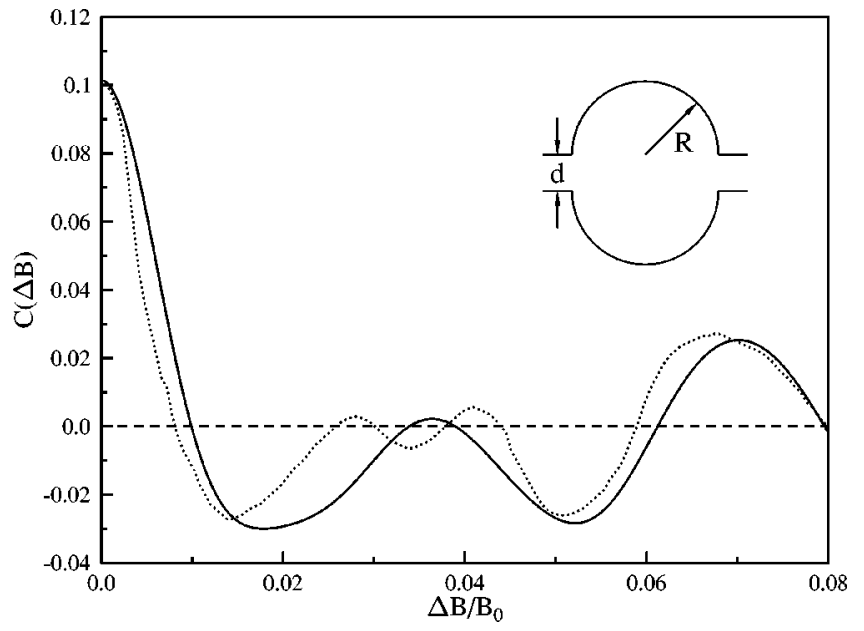

FIG. 9. Autocorrelation function for a stadium geometry with opposite leads (inset). Solid line: Fraunhofer diffraction approximation (FDA); dotted line, quantum calculation from Ref. 11. The semiclassical and quantum calculations are for $k_{F}=4.5 \pi / d, d$ $=R / 2, B_{0}=c k_{F} / d$.

perimental data, a full quantum calculation, and different semiclassical approximations for the stadium with different geometries. Experimental data of Marcus and co-workers ${ }^{2,19}$ are shown in Fig. 8 together with the semiclassical description in the Fraunhofer diffraction approximation (FDA) and the CTMC simulation for the stadium with leads at $90^{\circ}$ relative to each other. In Fig. 9, the semiclassical approximations are compared with full quantum-mechanical calculations ${ }^{11}$ for opposite leads. The full evaluation of the semiclassical expression within FDA [Eq. (38)] including off-diagonal correlations improves the agreement with the data and the quantum calculations compared to the CTMC evaluation. This is not surprising in view of the fact that the CTMC method is intrinsically a high-mode approximation while the experimental data and quantum calculations are for relatively low modes $(N \leqslant 3)$. Nevertheless, the oscillatory structures are qualitatively reproduced, thereby lending some credence to the intuitive path interference picture as the origin of the oscillatory structures. As will be discussed in the following section, however, this agreement, while not accidental, should be viewed with caution.

\section{VIOLATION OF UNITARY}

The quantum analog to the classical unitarity relation [Eq. (33)]

$$
\sum_{n}\left(T_{m n}+R_{m n}\right)=1
$$

imposes constraints on the fluctuations of the transmission and reflection probabilities,

$$
\delta T=-\delta R
$$

In other words, transmission and reflection are fully anticorrelated due to the unitarity of the $S$ matrix, or equivalently, due to flux conservation. Accordingly, the autocorrelation function for transmission $C^{(T)}$ and reflection $C^{(R)}$ should agree:

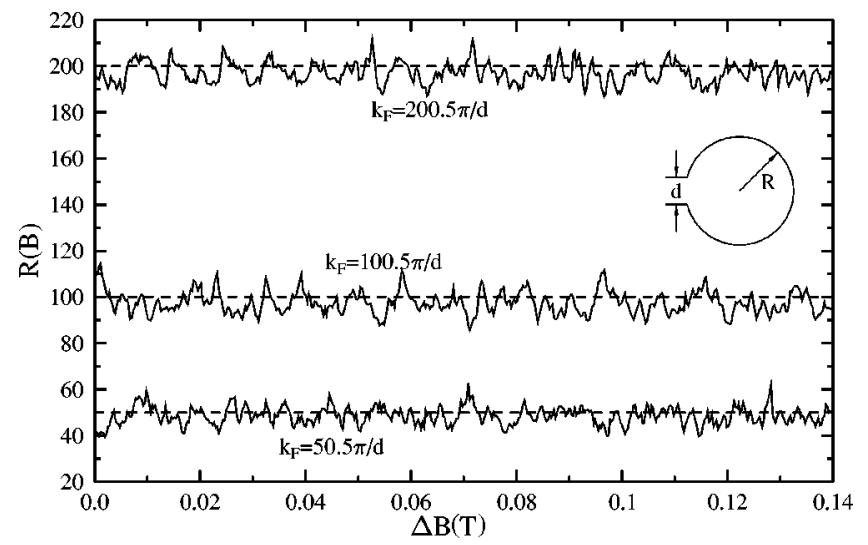

FIG. 10. $R^{(P S C)}(B)$ in a circle billiard with only one open lead for different $k_{F}$ and, therefore, different number of open leads $N$. $R=\sqrt{4 / \pi+1}, d=0.4$. The exact result, classically and quantum mechanically, is complete reflection: $R(B)=N$ (dashed line). The fluctuations demonstrate the violation of unitarity in the PSC approximation whose amplitude is independent of $N$.

$$
C^{(R)}(\Delta B)=C^{(T)}(\Delta B)
$$

While for quantum calculations on the one hand and classical calculations on the other hand Eq. (46) is satisfied, semiclassical approximations violate unitarity. Therefore, the equivalence of the correlation function in transmission and reflection is not guaranteed.

We present in the following a simple example that proves the fundamental deficiency of the semiclassical approach, which persists even when diffractive corrections are taken into account. To this end, we consider a circular billiard attached to only one lead. For this system, the sum over all paths can be easily performed. Moreover, with only one lead open, the quantum-mechanical and classical reflection probabilities are equal to one and $T_{m}=\delta T_{m}=0$. This means that the following equation should be true:

$$
\begin{aligned}
\delta R_{m}= & \frac{2 \pi}{(2 d)^{2}} \sum_{n} \sum_{q \neq q^{\prime}} \operatorname{sgn}(\bar{m}) \operatorname{sgn}(\bar{n}) \operatorname{sgn}\left(\bar{m}^{\prime}\right) \operatorname{sgn}\left(\bar{n}^{\prime}\right) \\
& \times\left|\widetilde{D}_{q} \widetilde{D}_{q^{\prime}}\right|^{1 / 2} \exp \left[i\left(\widetilde{S}_{q}-\widetilde{S}_{q^{\prime}}\right)-i \frac{\pi}{2}\left(\tilde{\mu}_{q}-\tilde{\mu}_{q^{\prime}}\right)\right]=0 .
\end{aligned}
$$

A numerical evaluation of Eq. (49) shows, however, that the sum over path pairs does not cancel in the semiclassical approximation. Figure 10 displays $R^{(P S C)}=\Sigma_{m} R_{m}^{(P S C)}$ as a function of the magnetic field for different $k_{F}$ (or equivalently, different $N$ ). The remarkable observation is that the fluctuations $\delta R^{(P S C)}$ that are due to the incomplete cancellation of the semiclassical path contributions are independent of $m$, i.e., they persist even for very high mode numbers. In other words, the limit $\delta R^{(P S C)}$ is not equal to zero, as $N$ goes to infinity $\left(\lim _{N \rightarrow \infty} \delta R^{(P S C)} \neq 0\right.$ ).

It is also instructive to study the influence of the lead width $d$ on $\delta R^{(P S C)}$. Figure 11 shows that the fluctuations for fixed $m=60$ and $k_{F}=100 \pi / d$ become smaller when the lead width is chosen larger. This explains the observation by Lin and Jensen ${ }^{14}$ who found that unitarity is approximately con- 


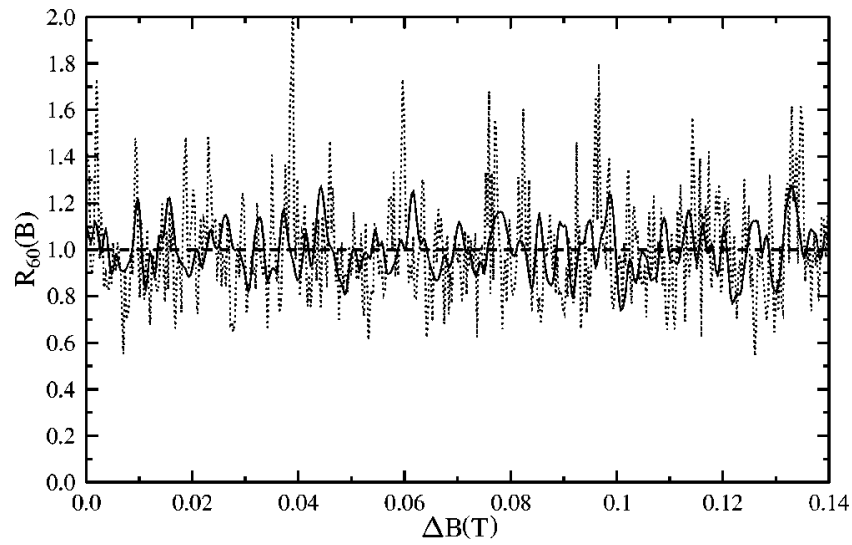

FIG. 11. $R_{m}^{(P S C)}(B)=\sum_{n} R_{m n}^{(P S C)}(B)$ for $m=60$ in the circle billiard with one open lead (inset of Fig. 10) at $k_{F}=100.5 \pi / d$, i.e., 100 open modes for two different choices of the lead width. Dotted line, $d=0.4$; solid line, $d=1.16$. The dashed line marks the exact result: $R_{m}(B)=1$. The violation of unitarity decreases for larger lead width.

served for a circle with wide leads. The increase of the violation of unitarity with smaller lead width (and therefore smaller de Broglie wavelength $\lambda \approx d / 2 m$ ) appears to contradict the notion that the semiclassical approximation should improve as $\lambda$ decreases. The origin for this behavior lies in the increase of the number of contributing bundles and stationary paths as the lead width decreases. Violation of unitarity is determined by the number of the terms in the path sum $\Sigma_{q \neq q^{\prime}}$ in Eq. (49). The larger the number of terms, the larger the fluctuation $\delta R^{(P S C)}$. In the present example, for $d=0.4$ the sum extends over 2174 paths while for $d=1.16$ only 1500 paths contribute. In fact, assuming the phases in Eq. (49) to be random, the RHS of the equation can be viewed as the realization of a random walk in the complex plane. Its end point will be further from the origin the more steps are taken. This problem persists when one employs the FDA rather than the PSC approximation. Figure 12 presents a comparison between those two approximations for $\delta R_{m}$ [Eq. (49)] with $m=10$. The deviation from unitarity are comparable for both semiclassical approximations. On the most fundamental level, the origin is the fact that for hard-wall structures the condition of a smooth potential on the scale of the de Broglie wavelength is locally not satisfied.

Another remarkable consequence of this failure is that the resulting autocorrelation function $C^{(R)}(\Delta B)$, which should vanish identically in the present case of only one open lead, displays an approximately Lorentzian shape according to Eq. (45). This is because the area distribution decays exponentially in this one-lead case as well. It has been argued that the "universal" approximation in terms of the squared Lorentzian would satisfy the condition of reciprocity between transmission and reflection. The present finding indicates, however, that the violation of unitarity may also affect the universal behavior, frequently assumed to be more robust against errors within semiclassical approximations.

The size of the unitarity fluctuations limits the region in which the semiclassical description of conductance fluctuations remains valid. The validity of the semiclassical description requires that the conductance fluctuations $\left\langle\delta T^{2}\right\rangle^{1 / 2}$ are larger than the mean deviation from unitarity. For the auto-

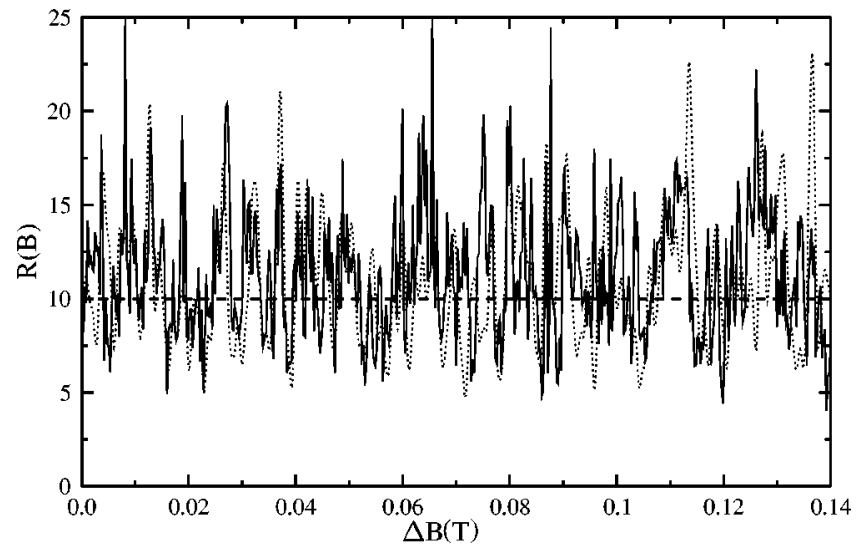

FIG. 12. Comparison of the deviation from unitarity between the PSC approximation (solid line) and the FDA (dotted line) in the circle with one open lead (inset of Fig. 10) at $k_{F}=10.5 \pi / d, d$ $=0.4$. The exact result is $R(B)=10$ (dashed line).

correlation function, this condition can be satisfied in general only near the first two maxima. Furthermore, if the mean values of $\langle T\rangle$ and $\langle R\rangle$ are very different as it can happen in regular structures, the range of the validity may be different in the reflection and transmission channels. Consequently, the range of validity of the semiclassical approximation for $C^{(T)}(\Delta B)$ and $C^{(R)}(\Delta B)$ will also be different.

\section{SUMMARY}

The semiclassical description of magnetoconductance fluctuations for ballistic microstructures involves directed areas enclosed by classical paths. We have developed a gaugeinvariant description of directed areas that can be visualized as the closure of areas by adding a virtual path to the real path connecting the leads. Gauge invariance of the resulting area distribution is found to be important for geometrysensitive nonuniversal properties of transport. We have calculated the magnetoconductance on different levels of semiclassical approximation, namely the Fraunhofer diffraction approximation, the primitive semiclassical approximation, and the classical trajectory Monte Carlo approximation. We find qualitative agreement with experimental data and full quantum calculations, which allows us to trace magnetoconductance fluctuations to path interferences. A fundamental limitation of semiclassical descriptions is the violation of unitarity in ballistic scattering. Deviations from unitarity can be even larger than the fluctuations itself. This poses limitations to the validity of the semiclassical description and points to the need to improve the semiclassical transport theory to a theory that takes into account nonclassical paths in the interior of the structure. ${ }^{30,31}$ Work along these lines is in progress.

\section{ACKNOWLEDGMENTS}

This work was supported by the NSF and U.S. DOE, Office of BES, Division of Chemical Sciences, under Contract No. DE-AC05-96OR22464 with Lockheed Martin Energy Research Corporation, and by the Austrian Science Foundation. 
${ }^{1}$ Quantum Transport in Ultrasmall Devices, Vol. 342 of NATO Advanced Study Institute, Series B: Physics, edited by D. K. Ferry, H. Grubin, C. Jacobini, and A.-P. Jauho (Plenum, New York, 1995).

${ }^{2}$ C. M. Marcus, A. J. Rimberg, R. M. Westervelt, P. F. Hopkins, and A. C. Gossard, Phys. Rev. Lett. 69, 506 (1992).

${ }^{3}$ M. W. Keller, O. Millo, A. Mittal, D. E. Prober, and R. N. Sacks, Surf. Sci. 305, 501 (1994).

${ }^{4}$ C. M. Marcus, A. J. Rimberg, R. M. Westervelt, P. F. Hopkins, and A. C. Gossard, Surf. Sci. 305, 480 (1994).

${ }^{5}$ J. P. Bird, K. Ishibashi, Y. Ochiai, M. Lakrimi, A. D. C. Grassie, K. M. Hutchings, Y. Aoyagi, and T. Sugano, Phys. Rev. B 52, 1793 (1993).

${ }^{6}$ I. V. Zozoulenko, R. Schuster, K.-F. Berggren, and K. Ensslin, Phys. Rev. B 55, 10209 (1997).

${ }^{7}$ D. K. Ferry, J. P. Bird, R. Akis, D. P. Pivin, Jr., K. M. Connolly, K. Ishibashi, Y. Aoyagi, T. Sugano, and Y. Ochiai, Jpn. J. Appl. Phys., Part 1 36, 3944 (1997).

${ }^{8}$ R. Akis, D. K. Ferry, and J. P. Bird, Phys. Rev. Lett. 79, 123 (1997).

${ }^{9}$ M. Gutzwiller, Chaos in Classical and Quantum Mechanics (Springer-Verlag, New York, 1991), and references therein.

${ }^{10}$ R. V. Jensen, Chaos 1, 101 (1991).

${ }^{11}$ R. A. Jalabert, H. U. Baranger, and A. D. Stone, Phys. Rev. Lett. 65, 2442 (1990).

${ }^{12}$ H. U. Baranger, R. A. Jalabert, and A. D. Stone, Chaos 3, 665 (1993).

${ }^{13}$ W. A. Lin, J. B. Delos, and R. V. Jensen, Chaos 3, 655 (1993).
${ }^{14}$ W. A. Lin and R. V. Jensen, Phys. Rev. B 53, 3638 (1996).

${ }^{15}$ W. A. Lin, Chaos Solitons Fractals 8, 995 (1997).

${ }^{16}$ H. Ishio and J. Burgdörfer, Phys. Rev. B 51, 2013 (1995).

${ }^{17}$ C. D. Schwieters, J. A. Alford, and J. B. Delos, Phys. Rev. B 54, 10652 (1996).

${ }^{18}$ L. Wirtz, J.-Z. Tang, and J. Burgdörfer, Phys. Rev. B 56, 7589 (1997).

${ }^{19}$ J. Z. Tang, L. Wirtz, J. Burgdörfer, and C. M. Marcus, Phys. Rev. B 57, 9875 (1998).

${ }^{20}$ R. Landauer, IBM J. Res. Dev. 1, 223 (1957).

${ }^{21}$ H. U. Baranger and A. D. Stone, Phys. Rev. B 40, 8169 (1989).

${ }^{22}$ W. H. Miller, Adv. Chem. Phys. 25, 69 (1974).

${ }^{23}$ R. L. Schult, H. W. Wyld, and R. G. Ravenhall, Phys. Rev. B 41, 12760 (1990).,

${ }^{24}$ L. A. Bunimovich, Funct. Anal. Appl. 8, 254 (1974); G. Benettin and J. M. Strelcyn, Phys. Rev. A 17, 773 (1978).

${ }^{25}$ R. Abrines and I. C. Percival, Proc. Phys. Soc. London 88, 861 (1966).

${ }^{26}$ C. O. Reinhold and C. A. Falcón, Phys. Rev. A 33, 3859 (1986).

${ }^{27}$ X. Yang, H. Ishio, and J. Burgdörfer, Phys. Rev. B 52, 8219 (1995).

${ }^{28}$ M. Schreier, K. Richter, G.-L. Ingold, and R. A. Jalabert, Eur. Phys. J. B 3, 387 (1998).

${ }^{29}$ C. K. Beenakker, Rev. Mod. Phys. 69, 731 (1997), and references therein.

${ }^{30}$ J. B. Keller, J. Opt. Soc. Am. 52, 116 (1962).

${ }^{31}$ G. Vattay, J. Cserti, G. Palla, and G. Szalka, Chaos Solitons Fractals 8, 1031 (1997). 